$$
\text { CONF - } 970503--270
$$

\title{
ANALYSIS AND APPLICATION OF MICROWAVE RADIATION FROM THE DAMPING MANIFOLDS OF THE SLAC DAMPED DETUNED STRUCTURES (DDS) ${ }^{1}$
}

\author{
RECEIVED
JULOT 997
OSTI
ECENE
OST 999
STI \\ R.M. Jones ${ }^{\dagger}$, M. Seidel ${ }^{\dagger}$, N.M. Kroll ${ }^{\dagger}$, R.H. Miller ${ }^{\dagger}$, \\ C. Adolphsen ${ }^{\dagger}$, K.L.F. Bane, R.D. Ruth ${ }^{\dagger}$, and J. Wang ${ }^{\dagger}$ \\ $\dagger$ Stanford Linear Accelerator Center, M/S 26, \\ P.O Box 4349, Stanford, CA 94309 \\ $\ddagger$ University of California, San Diego, \\ La Jolla, CA 92093-0319
}

\begin{abstract}
The power spectrum emerging from the damping manifolds of a DDS provides valuable quasi-local information on the displacement of a drive beam from the axis of individual cells, where the displacement may be due to beam offset, small cell misalignment, or a combination of the two. The degree of localization and the indexing of frequency to cell number is determined directly from the spectral function theory. Examples for specific DDS designs will be presented. These relations can be used to determine geometrical misalignment patterns.
\end{abstract}

\section{DISCLAIMER}

This report was prepared as an account of work sponsored by an agency of the United States Government. Neither the United States Government nor any agency thereof, nor any of their employees, makes any warranty, express or implied, or assumes any legal liability or responsibility for the accuracy, completeness, or usefulness of any information, apparatus, product, or process disclosed, or represents that its use would not infringe privately owned rights. Reference herein to any specific commercial product, process, or service by trade name, trademark, manufacturer, or otherwise does not necessarily constitute or imply its endorsement, recommendation, or favoring by the United States Government or any agency thereof. The views and opinions of authors expressed herein do not necessarily state or reflect those of the United States Government or any agency thereof.

\section{Paper presented at the 1997 Particle Accelerator Conference \\ Vancouver, British Columbia, Canada \\ May 12-16, 1997}

\footnotetext{
${ }^{1}$ Supported by Department of Energy grant number DE-FG03-93ER40759 and DE-AC03-76SF00515 ${ }^{\dagger}$
} 


\title{
ANALYSIS AND APPLICATION OF MICROWAVE RADIATION FROM THE DAMPING MANIFOLDS OF THE SLAC DAMPED DETUNED STRUCTURES (DDS)
}

\author{
R.M. Jones ${ }^{\dagger}$, M. Seidel ${ }^{\dagger}$, N.M. Kroll ${ }^{\dagger \ddagger}$, R.H. Miller ${ }^{\dagger}$, \\ C. Adolphsen ${ }^{\dagger}$, K.L.F. Bane, R.D. Ruth ${ }^{\dagger}$, and J. Wang ${ }^{\dagger}$ \\ $\uparrow$ Stanford Linear Accelerator Center, M/S 26, P.O Box 4349, Stanford, CA 94309 \\ †University of California, San Diego, La Jolla, CA 92093
}

\begin{abstract}
The power spectrum emerging from the damping manifolds of a DDS provides valuable quasi-local information on the displacement of a drive beam from the axis of individual cells, where the displacement may be due to beam offset, small cell misalignment, or a combination of the two. The degree of localization and the indexing of frequency to cell number is determined directly from the spectral function theory. Examples for specific DDS designs will be presented. These relations can be used to determine geometrical misalignment patterns.
\end{abstract}

\section{INTRODUCTION}

The damped detuned structure (DDS) is a detuned accelerator structure [1] to which four waveguide like structures have been attached in an azimuthally symmetric manner. These "waveguides", called damping manifolds by convention, are extended along the length of the accelerator structure and are coupled to all cells except 3 at either end. These damping manifolds are intended to perform three functions: 1 . provide moderate damping of dipole modes excited by an offset drive beam by conducting their energy to loads at the end of the structure, 2 . serve as pumping manifolds for the accelerator cells, and 3. provide via the power spectrum of radiation from the ends of the manifolds, beam position and structure alignment information [2]. In this paper we focus on the third of the above and in particular extend the preliminary analysis reported in [3] on power spectrum localization and its application to the determination of structure misalignment from power spectrum measurements. The appliction of manifold radiation to beam positioning is discussed in [4] and [5].

\section{THE MANIFOLD POWER SPECTRUM AND ITS RELATION TO OFFSET LOCATION}

In the independent cell model (often referred to as the uncoupled model) [1] one treats the cells as independent entities which respond in a narrow band about their synchronous frequencies to an offset velocity $c$ particle. Thus the appearance of a frequency band in the power spectrum of the manifold radiation is associated with offsets at the cells which respond at frequencies within that band. The underlying idea behind this model is the expectation that the structure in the vicinity of a particular cell is sufficiently like a uniform structure based upon that cell that the concept of the cell synchronous frequeticy is applicable. Since the establishment of synchronism must involve several cells, one expects even in the independent cell model that the association of cell location with radiation frequency must have some width. In this section we apply the equivalent circuit theory, including detuning, damping, and manifold reflections to a determination of this relation.

The power spectrum for a beam with uniform offset $\delta \mathrm{x}$ is proportional to $\mathrm{P}(\mathrm{f})$ given below by [3]:

$$
P_{u}(f)=\delta x^{2} f \sin \phi(f)|A(f) /(1+R(f))|^{2}
$$

Here $\phi(f)$ is the manifold phase advance function, $R(f)$ the reflection amplitude, and $A(f)$ the manifold excitation amplitude for unit offset [6] all evaluated at the terminating manifold section, and $f$ is the frequency. This function computed for DDS 1 is shown in Fig. 1 and compared with the results from the ASSET experiment [4].

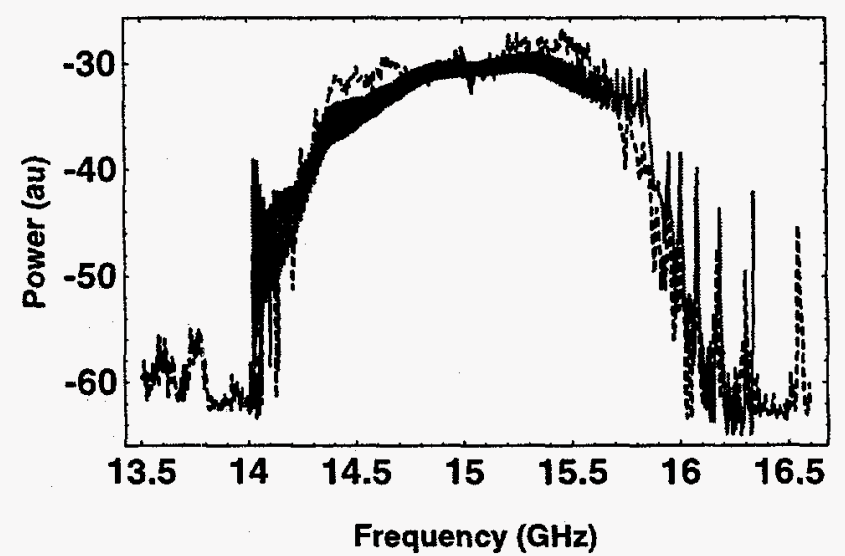

Fig 1: Power spectrum radiated to the downstream HOM coupler: experimental values are shown dashed and those calculated from circuit theory are indicated by the solid curve. 


\section{DISCLADMER}

Portions of this document may be illegible in electronic image products. Images are produced from the best available original document. 
In the equivalent circuit theory both the cell and manifold amplitudes are linearly related to the excitations at the individual cells so that instead of defining $A(f)$ for uniform offset we write $A(f)=\sum u_{n}(f) \delta x_{n}$ or with $M_{n}(f)=\sqrt{f \sin \phi(f)} u_{n}(f) /(1+R(f))$ :

$$
P_{u}(f)=\left|\sum M_{n}(f) \delta x_{n}\right|^{2}
$$

The $u_{n}(f)$ may be determined from the procedure outlined in [3]. To apply (2.2) to the localization problem we set $\delta \mathrm{x}_{\mathrm{n}}=\delta \mathrm{x}$ for a set of adjacent cells $\mathrm{n}-\mathrm{r}$ to $\mathrm{n}+\mathrm{r}$ and zero elsewhere and compute the power spectrum. The result for a representaive set of central cells is shown in Fig. 2. The pair of integers above each sharp peak in the figure give the central cell number $n$ on the left and the span specifier $r$ on the right. The number $r$ is determined by requiring it to be the smallest number which gives a peak equal in amplitude to that obtained by applying $\delta x$ to all of the cells. Because $r$ is a small integer, this criterion is not met exactly, but the figure indicates how well it is met. We call the central frequency of the peaks the coupled synchronous frequency of the central cell.

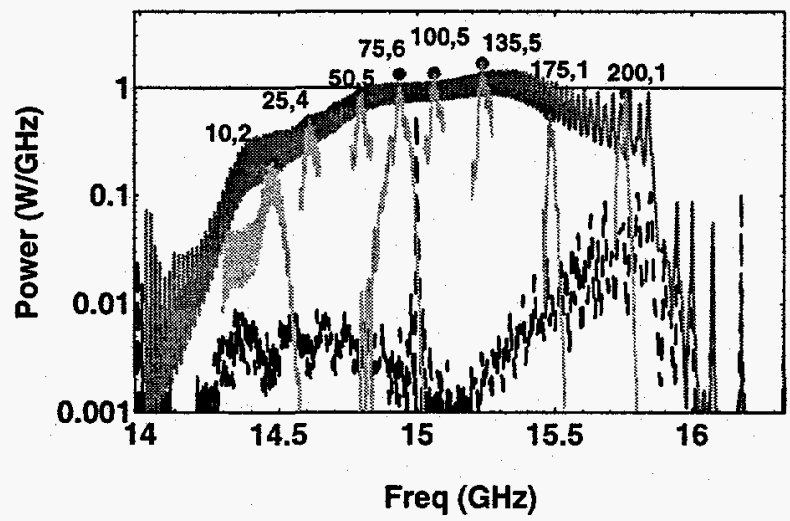

Fig 2: Power spectrum emitted from the downstream DDS manifold illustrating the narrow frequency bands emitted by localized offsets. The pairs of numbers above each peak indicate the central cell and the span specifier (see text) of the localized offsets.

The curve of the coupled synchronous frequency in Fig. 3 is a fit to the points determined in Fig. 2; the horizontal bars represent a fit to the span. The synchronous frequencies of the independent cell model (called "uncoupled") are shown for comparison, and one notes a shift, small, but significant for locating misalignments. The span $2 r+1$ varies from three to thirteen cells and is largest at frequencies where the mode are most extended. Almost all the (damping suppressed) modes of DDS 1 are standing waves terminating within the structure, but their extension varies from one or two cells to over a hundred. For the more extended modes the excitation region is well localized within the mode.

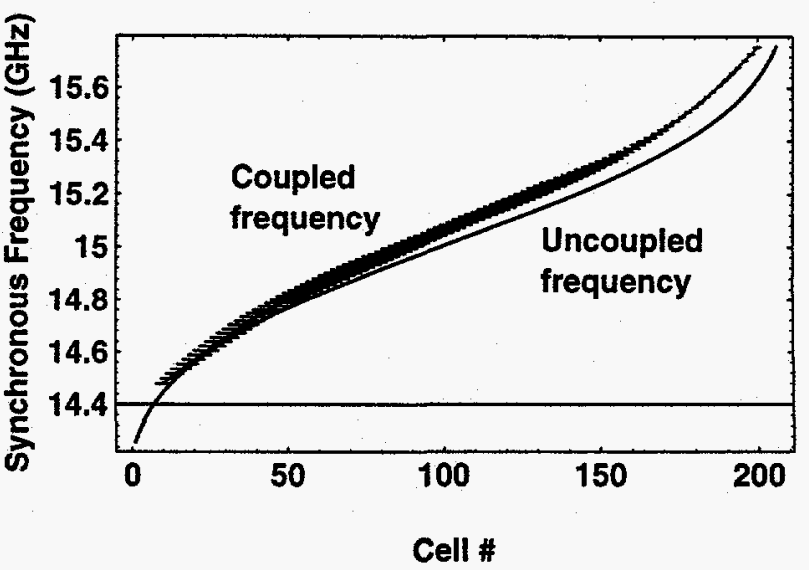

Fig 3: Illustration of the deviation of the synchronous frequency from the uncoupled one due to cell-to-cell detuning. The short horizontal lines indicate the extent to which cell offsets may be localized by frequency.

\section{BEAM-BASED STRUCTURE ALIGNMENT}

In fabricating the first DDS a number of cell misalignments occurred, the worst being 60 microns at cells 45 to 46 . This is illustrated in Fig. 4 in which the second and third of the three mechanical measurements performed using a $\mathrm{CMM}$ (coordinate measuring machine) are plotted. The third measurement was performed after the ASSET experiment. However, it is evident that the absolute value of the jog at cell 45 is similar to data set 2 . Indeed the main difference between the two data sets is an angular shift, a translational offset, and a bend.

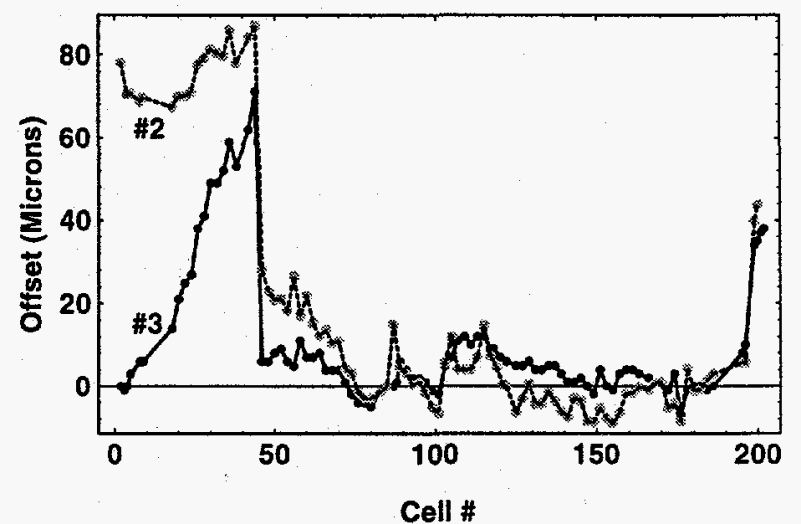

Fig 4: Two set of mechanical alignment data: measured before the ASSET experiment (data set 2) and after the completion of the experiment (data set 3 )

A beam based alignment measurement carried out during the ASSET experiment is reported in [5]. The measurement, performed by stepping the beam transversely across the structure, provided a complete power spectrum at each step. From this data one obtains the beam position which minimizes the power at each frequency and generates the curve of minimum power position versus frequency given in [5]. A curve of minimum power position versus cell number can be 
obtained by identifying the frequency with the coupled synchronous frequency given in Fig. 3 as a function of cell number. One can then relate this measurement to structure misalignment by assuming that when the power is minimized at a particular coupled synchronous frequency that the beam is centered on the cell to which that frequency belongs. The cell offsets obtained in this way (i.e. remapping) are, after translational and angular adjustment, compared to the two sets of CMM data in Figs. 5 and 6 . The adjustments are legitimate because the position and tilt of the beam relative to the axis of the CMM measurements is not known.

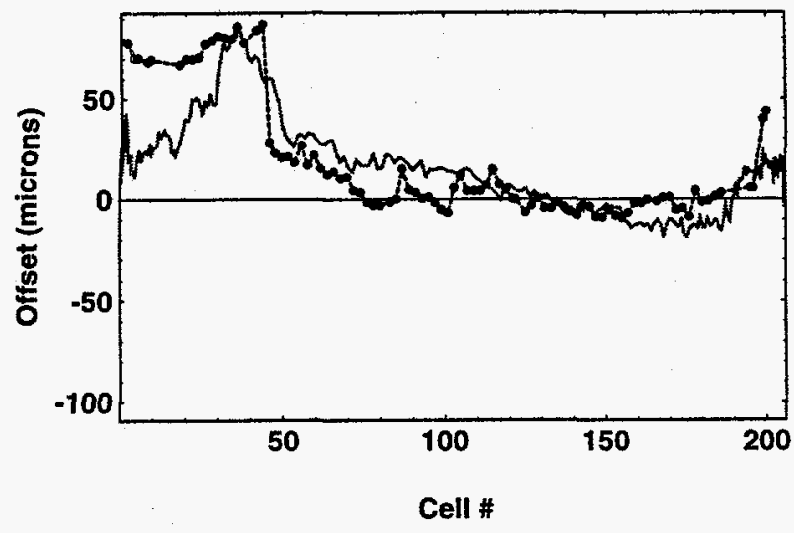

Fig 5: Comparison of the CMM data set \# 2 versus the ASSET power minimization position data remapped from frequency to cell number (see text).

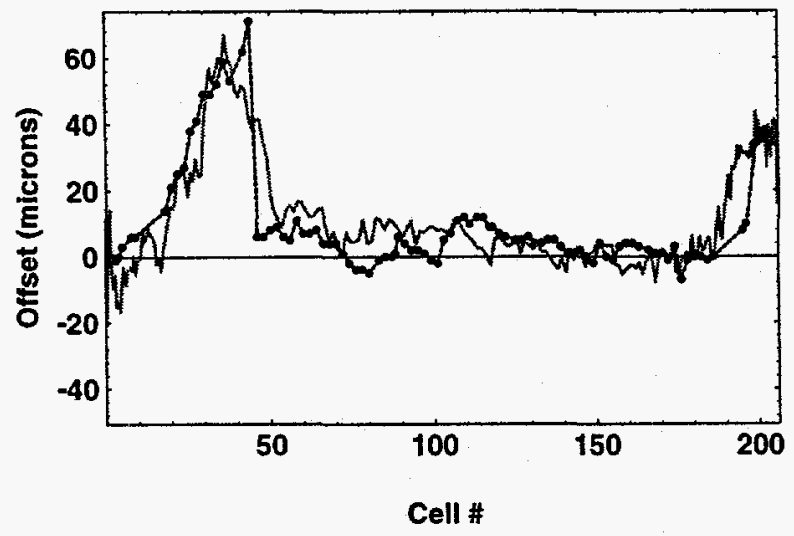

Fig 6: Comparison of the CMM data set \# 3 versus the ASSET power minimization position data remapped from frequency to cell number (see text).

The minimum power position $\mathrm{X}(\mathrm{f})$ can also be linearly related to the mechanical offsets by writing $\delta x_{n}$ in Eq. (2.2) as $X(f)-x_{n}$ where $x_{n}$ is the mechanical offset of the $n$ 'th cell and minimizing (2.2) with respect to $X(f)$. In terms of 295 discrete frequencies $f_{n}$ for which the measurements were made one finds:

$$
X\left(f_{m}\right)=\sum_{n} K_{n}\left(f_{m}\right) x_{n}
$$

where:

$$
\mathrm{K}_{\mathrm{n}}(\mathrm{f})=\operatorname{Re}\left\{\sum_{m} \mathrm{M}_{\mathrm{m}}^{*}(\mathrm{f}) \mathrm{M}_{\mathrm{n}}(\mathrm{f})\right\} /\left|\sum_{m} \mathrm{M}_{\mathrm{m}}(\mathrm{f})\right|^{2}
$$

It is thus a set of linear equations for 295 power minimization positions in terms of 206 offsets. The determination of the $X\left(f_{m}\right)$ from a set of $x_{n}$ is straightforward and reported in [3]. For comparison with the remapping method described above, however, one needs to determine the cell offsets from the minimum power positions. Equation (3.1) is solved for the $x_{n}$ by employing the pseudo (ie Moore-Penrose) inverse, based upon the singular value decomposition of $\mathrm{K}_{n}\left(\mathrm{f}_{\mathrm{w}}\right)$ regarded as a $295 \times 206$ matrix. The result obtained (after stabilization by application of a tolerance criterion on the singular values and Savitzky-Golay filtering on the output) is shown in Fig. 7, where it is compared to that obtained by remapping. The agreement is satisfactory, but there is no evidence that the pseudo inverse method improves the delineation of abrupt offset changes. Thus for the present at least we consider the remapping method to be preferred, both because it is simpler and because it does not involve uncertain numerical procedures.

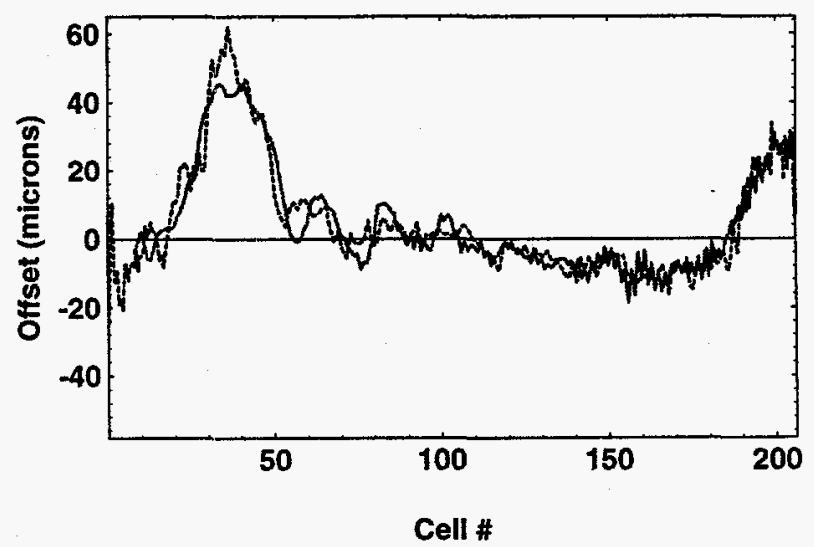

Fig 7: Cell offsets computed by the pseudo inverse method(solid curve) compared to those computed by remapping as in Figs. 5 and 6 (dashed curve).

\section{ACKNOWLEDGMENTS}

This work is supported by DOE grant number DE-FG0393ER 40759 $9^{\ddagger}$ and DE-AC03-76SF00515 ${ }^{\dagger}$. We have benefited greatly from discussions at the weekly structures meeting at SLAC, where these results were first presented and thank all members of the group.

\section{REFERENCES}

[1] K. Bane et al, Part. Accel. 42, p 123 (1993)

[2] N. Kroll et al, AIP Proc. 335, p 777 (1994)

[3] R.M. Jones et al, Analysis ... Manifold Radiation ...,

Proc. 7'th Workshop on Advanced Accelerator Concepts,

[4] M. Seidel et al, Proc. Linac96 p 653 (1996)

[5] M. Seidel, Studies .. of Dipole Mode .., 5B3 Pac'97

[6] R.M. Jones et al, Proc. EPAC 96, p 1292 (1996) 